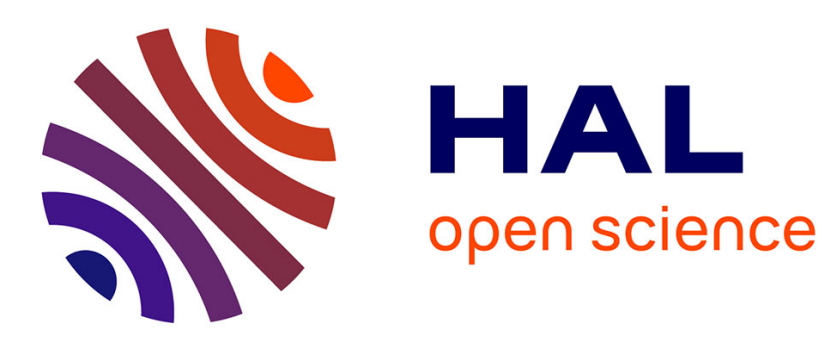

\title{
Adaptive state estimation for a class of uncertain nonlinear systems with output time-delays
}

Habib Dimassi, Antonio Loria, Safya Belghith

\section{To cite this version:}

Habib Dimassi, Antonio Loria, Safya Belghith. Adaptive state estimation for a class of uncertain nonlinear systems with output time-delays. Decision and Control (CDC), 2012 IEEE 51st Annual Conference on, Jun 2012, United States. pp.2346-2351, 10.1109/CDC.2012.6426865 . hal-00831386

\section{HAL Id: hal-00831386 https://hal.science/hal-00831386}

Submitted on 6 Jun 2013

HAL is a multi-disciplinary open access archive for the deposit and dissemination of scientific research documents, whether they are published or not. The documents may come from teaching and research institutions in France or abroad, or from public or private research centers.
L'archive ouverte pluridisciplinaire HAL, est destinée au dépôt et à la diffusion de documents scientifiques de niveau recherche, publiés ou non, émanant des établissements d'enseignement et de recherche français ou étrangers, des laboratoires publics ou privés. 


\title{
Adaptive state estimation for a class of uncertain nonlinear systems with output time-delays
}

\author{
Habib Dimassi, Antonio Loría, Safya Belghith
}

\begin{abstract}
In this paper, we propose an adaptive observer for nonlinear systems with slope restricted nonlinearities, unknown parameters and delayed outputs. The delay is assumed constant and the unknown parameter is assumed piece-wise constant. Based on the Lyapunov-krasovskii approach, we show that, for sufficiently small values of the time-delay, both state estimation and parametric convergence are ensured under a condition of persistent excitation. The result is illustrated via two numerical examples.
\end{abstract}

\section{INTRODUCTION}

Time-delays occur frequently in many engineering applications such that communication systems, biochemical reactors, mechanical systems and many other applications. In this context, the issue of state estimation of systems with time-delays which may appear in states, inputs or outputs has received increasing attention. For instance, in [1], the problem was considered for the case of only delayed states and in [2] the authors investigate the case of delayed inputs. Particularly, the problem of observer design for nonlinear systems with delayed outputs has attracted specific attention in the last decade. The existing approaches in the recent literature extend principally the available results on the nonlinear observer design to the case of delayed outputs; however, there exists only a countable number of publications investigating this particular issue and it remains many possible extensions to be investigated. For instance, in the reference [3], based on the design of state observers from a drift-observability property, the authors has presented a solution for the case of delayed outputs by proposing a chain of observation algorithms which ensure global exponential convergence of the estimation error. A similar conceptually design method has been adopted in [4], however the proposed nonlinear observer possesses a state-dependent gain which is computed from the solution of a system of firstorder singular partial differential equations and the conditions ensuring the convergence of the observation error were given. In [5], the authors propose two cascaded observers to reconstruct the system states of a linear time invariant system with delayed measurements where the time-delay is assumed a known piece-wise function of time. On the other hand, in [6], the authors propose a nonlinear observer for a class of drift observable nonlinear systems with a bounded

CNRS, LSS-Supelec, 3 Rue Joliot-Curie, 91192, Gif/Yvette, France; Sys'com-Ecole Nationale d'Ingénieurs de Tunis (ENIT), Tunisia. dimassielss.supelec.fr; loriallss.supelec.fr; safya.belghith@enit.rnu.tn. time varying observation delay. Based on the LyapunovRazumikhiin approach, asymptotic and exponential convergence of the estimation error have been proved to be guaranteed under some conditions. More recently, in the references [7],[8]; an elegant approach based on high gain observer design for a class of triangular nonlinear systems with respectively constant and time varying delayed measurement has been proposed and asymptotic convergence was shown to be ensured by choosing a suitable Lyapunov-Krasovskii functional if some condition relating the observer gain and the upper bound of the time delay is satisfied.

On the other hand, the problem of joint state and unknown parameters estimation using adaptive observers has been widely investigated in the literature for the cases of linear and nonlinear systems. For instance, in [9], the author introduces a unifying adaptive observer form for nonlinear systems under some additional "passivity-like" condition on the observation error. Another solution to the adaptive estimation problem was also proposed in [10] for the case of a class of MIMO linear time-varying systems. This approach has been combined with high gain observer design in [11] to reconstruct a new adaptive observer for a class of single output uniformly observable systems. More recently, in [12], the authors address the problem of the adaptive observer design for a class of nonlinear time-varying systems with parametric uncertainties in the context of synchronization of chaotic systems by exploiting the persistent excitation property of chaotic systems.

Motivated by the design approach developed in the references [7],[8], we propose in this paper a new adaptive observer tailored for a class of nonlinear systems with slope restricted nonlinearities, unknown parameters (the regressors are also assumed slope restricted) and delayed outputs. To the best of our knowledge, adaptive state estimation in such scenario is not solved yet, however this problem is motivated by many applications where modeling errors, uncertainties and measurement delays occur simultaneously. Furthermore, chaotic communication under transmission delays is an important application of the considered scenario in this paper.

Based on the Lyapunov-Krasovskii approach, we show that for sufficiently small values of the time-delay, both state estimation and parametric convergence are ensured under a condition of persistent excitation. The paper is organized as follows. In the following section, we motivate and formulate the problem. In section III, we present the adaptive observer and analyze both state and parameter convergence. In section IV, we show the effectiveness of the proposed approach via two numerical examples. Finally, some con- 
cluding remarks will be given in section $\mathrm{V}$.

Notation. The following notation is used throughout this paper. $|\cdot|$ denotes the absolute value for scalars, the Euclidean norm for vectors, and the induced norm for matrices. $I$ represents the identity matrix. The smallest and largest eigenvalues of $S$ are denoted by $s_{m}$ and $s_{M}$ respectively.

\section{PROBLEM STATEMENT}

In this paper, we address the problem of observer design for the following class of nonlinear systems

$$
\begin{aligned}
\dot{x} & =A x+F f(H x, u)+B \sum_{k=1}^{q} \Psi^{k}\left(R_{k} x, u\right) \theta_{k} \\
y & =C x(t-h)
\end{aligned}
$$

where $x \in \mathbb{R}^{n}$ denotes the state vector, $u \in \mathbb{R}^{l}$ denotes the input vector and $y \in \mathbb{R}^{p}$ represents the output vector subject to the observation delay $h$. It is assumed that the delay $h$ is known and constant. $A \in \mathbb{R}^{n \times n}, B \in \mathbb{R}^{n \times s}$, $F \in \mathbb{R}^{n \times m}, C \in \mathbb{R}^{p \times n}$ and $H=\left(H_{1}, H_{2}, \ldots, H_{m}\right)^{T} \in$ $\mathbb{R}^{m \times n}$ and $R_{k}=\left(R_{k 1}, \ldots, R_{k s}\right)^{T} \in \mathbb{R}^{s \times n}$ are constant matrices; with $H_{i}$ is the $i-t h$ raw of $H$; for $i=1 . . m$ and $R_{k_{i}}$ is the $i-t h$ raw of $R_{k}$; for $i=1 . . s \mathrm{We}$ define $\theta:=\left(\theta_{1}, \ldots, \theta_{q}\right)^{T}$ the unkown parameters vector, where $\theta_{k}(t) \in \mathbb{R}$ are assumed piece-wice constant. $f: \mathbb{R}^{m} \times \mathbb{R}^{l} \rightarrow \mathbb{R}^{m}$ is a nonlinear function such that $f(H x, u)=\left(f_{1}\left(H_{1} x, u\right), \ldots, f_{m}\left(H_{m} x, u\right)\right)$ and $\Psi^{k}: \mathbb{R}^{s} \times$ $\mathbb{R}^{l} \rightarrow \mathbb{R}^{s}$ represent persistently exciting functions such that $\Psi^{k}\left(R_{k} x, u\right)=\left(\Psi_{1}^{k}\left(R_{k 1} x, u\right), \ldots, \Psi_{s}^{k}\left(R_{k s} x, u\right)\right)$.

We assume that $\forall i \in 1 . . m, f_{i}(\cdot)$ satisfies the following slope restriction condition,

$\forall u \in \mathbb{R}^{l}, \forall \xi_{1}, \xi_{2} \in \mathbb{R}$ such that $\xi_{1} \neq \xi_{2}$, we have the following

$$
0 \leq \frac{f_{i}\left(\xi_{1}, u\right)-f_{i}\left(\xi_{2}, u\right)}{\xi_{1}-\xi_{2}} \leq b
$$

Similiary, we assume that $\Psi^{k}\left(R_{k} x, u\right)$ satisfy the same properties of $f(H x, u)$ detailed above such that the slope is restricted in $\left[0, \bar{b}_{k}\right]$, with $k=1 . . q$.

The addressed plant in this paper is motivated by many systems and applications subject to the slope restricted nonlinearities (2) such as mechanical systems (e.g. active magnetic bearing model), in robotics (e.g. robot arm with flexible joint), chaotic systems (e.g. Chua, Duffing, Van der Pol..), etc. We investigate in this paper the problem of joint state and unknown parameters in the presence of measurement delays which occur frequently in practice. To the best of our knowledge, the problem of observer design was solved only in the presence of either unknown parameters or measurement delays, but not simultaneously. We note also that an important application of the addressed problem is the synchronization of chaotic systems for the transmission of informations under communication delays - see the illustrative example in section IV-B.

In summary, our objective is to design a dynamical system (adaptive observer) which estimates the state $x(t)$ and the unknown parameter $\theta(t)$ of the considered nonlinear system (1) despite the time-delay affecting the measured output.

\section{MAIN RESULT}

The problem of observer design for nonlinear systems with slope restricted nonlinearities in the case of non delayed outputs was investigated in the last decade and particularly by Arcak and Kokotović who introduced, in [13], an approach making use of the bounds of the nonlinear term slope to prove the convergence of the estimation error. On the other hand, the problem of joint state and unknown parameters estimation using adaptive observers in the case of non delayed outputs was investigated in the literature -see [9], [10]. Motivated by the nonlinear observers for systems with slope restricted nonlinearities and adaptive-observers design, we propose the following adaptive observer:

$$
\begin{aligned}
\dot{z}= & A z+F f(H z, u)+B \sum_{k=1}^{q} \Psi^{k}\left(R_{k} z, u\right) \hat{\theta}_{k} \\
& +K(\hat{y}-C z(t-h)) \\
\dot{\hat{\theta}}_{k}= & \rho \Upsilon_{k}^{-1} \Psi^{k}\left(R_{k} z, u\right)^{T} M(y(t)-C z(t-h)) \\
\dot{\Upsilon}_{k}= & -\alpha \Upsilon_{k}+\Psi^{k}\left(R_{k} z, u\right)^{T} B^{T} B \Psi^{k}\left(R_{k} z, u\right) \\
\Upsilon_{k}(0)> & 0, \quad k=1 . . q .
\end{aligned}
$$

where $z(t)$ and $\hat{\theta}_{k}(t)$ denote respectively the estimated state and the estimated parameters; $\rho$ and $\alpha$ are positive constants.

We define the observation error $e(t):=x(t)-z(t)$ and the adaptation error $\tilde{\theta}(t):=\left(\tilde{\theta}_{1}(t), \ldots, \tilde{\theta}_{q}(t)\right)$, where $\tilde{\theta}_{k}(t):=$ $\theta_{k}(t)-\hat{\theta}_{k}(t)$, for $k=1 . . q$.

Using the fact that $\dot{\theta}_{k}(t)=0$ almost every where, the error system is described by the following equations

$$
\begin{aligned}
\dot{e}= & A e-K C e(t-h)+F \eta(H e, x, u) \\
& +B \sum_{k=1}^{s}\left(\bar{\eta}^{k}\left(R_{k} e, x, u\right) \theta_{k}+\Psi^{k}\left(R_{k} z, u\right) \tilde{\theta}_{k}\right)(4 \mathrm{a}) \\
\dot{\tilde{\theta}}_{k}= & -\rho \Upsilon_{k}^{-1} \Psi^{k}\left(R_{k} z, u\right)^{T} M C e(t-h),
\end{aligned}
$$

where $\eta(H e, x, u)=\left(\eta_{1}\left(H_{1} e, x, u\right), \ldots, \eta_{m}\left(H_{m} e, x, u\right)\right)$ such that for $i=1 . . m$,

$\eta_{i}\left(H_{i} e, x, u\right)=f_{i}\left(H_{i} x, u\right)-f_{i}\left(H_{i} x-H_{i} e, u\right)$;

and $\bar{\eta}^{k}\left(R_{k} e, x, u\right)=\left(\bar{\eta}_{1}^{k}\left(R_{1} e, x, u\right), \ldots, \bar{\eta}_{s}^{k}\left(R_{s} e, x, u\right)\right.$ such that for $i=1 . . s$,

$\bar{\eta}_{i}^{k}\left(R_{k i} e, x, u\right)=\Psi^{k}\left(R_{k i} x, u\right)-\Psi^{k}\left(R_{k i} x-R_{k i} e, u\right)$.

We initialize the estimation error equation (4a) as

$$
e(\vartheta)=\phi(\vartheta), \quad \forall-h \leq \vartheta \leq 0 .
$$

where $\phi(\cdot)$ is a continuous function defined in the interval $[-h, 0]$.

Now, in view of the slope restriction (2) with $\xi_{1}=H_{i} x$ and $\xi_{2}=H_{i} x-H_{i} e, \forall e \neq 0, \forall x, \forall u$

$$
0 \leq \frac{\eta_{i}\left(H_{i} e, x, u\right)}{H_{i} e} \leq b .
$$

Multiplying on both sides of (6) by $\eta_{i}\left(H_{i} e, x, u\right) H_{i} e$, we get: for $i=1 . . m, \forall t \geq 0, \forall e, \forall x, \forall u$

$$
\eta_{i}\left(H_{i} e, x, u\right)\left[\eta_{i}\left(H_{i} e, x, u\right)-b H_{i} e\right] \leq 0
$$


which means that $\eta(H e, x, u)$ belongs to the sector $[0, \mathrm{~b}]$. Let $D=\operatorname{diag}\left(d_{1}, d_{2}, \ldots, d_{m}\right)$ with $d_{i}>0, \forall i=1 . . m$,. After some manipulations, one can easily obtain the following relation

$$
\begin{array}{r}
-\eta^{T}(H e, x, u) D \eta(H e, x, u) \\
+b \eta(H e, x, u) D H e \geq 0 .
\end{array}
$$

Proceeding with the same reasoning for $\Psi^{k}\left(R_{k} x, u\right)$, we obtain the following: $\forall k=1 . . q$ and $\bar{D}_{k}=\operatorname{diag}\left(\bar{d}_{k 1}, \ldots, \bar{d}_{k s}\right)$ with $\bar{d}_{k i}>0, \forall i=1 . . s$.

$$
\begin{array}{r}
-\bar{\eta}^{k T}\left(R_{k} e, x, u\right) \bar{D}_{k} \bar{\eta}\left(R_{k} e, x, u\right) \\
+b \bar{\eta}^{k}\left(R_{k} e, x, u\right) \bar{D}_{k} R_{k} e \geq 0
\end{array}
$$

Assumption 1: We assume that there exist a symmetric positive definite matrix $P$, diagonal positive matrices $D$, $\bar{D}_{1}, \ldots, \bar{D}_{q}$; regular matrices $M$ and $K$ of appropriate dimensions and a positive constant $\varepsilon$ such that

$$
\begin{gathered}
\mathcal{S}=\left[\begin{array}{ccc}
-Q+\varepsilon I & P F+b H^{T} D & \Lambda \\
F^{T} P+b D H & -2 D & 0 \\
\Lambda^{T} & 0 & -2 \bar{D}
\end{array}\right] \leq 0 \\
B^{T} P=M C
\end{gathered}
$$

where $Q=-\left[(A-K C)^{T} P+P(A-K C)\right]$, $\Lambda=\left(P B+\bar{b}_{1} R_{1}^{T} \bar{D}_{1}, \cdots, P B+\bar{b}_{s} R_{s}^{T} \bar{D}_{s}\right)$ and $\bar{D}=\operatorname{block}-\operatorname{diag}\left(\bar{D}_{1}, \ldots, \bar{D}_{s}\right)$

Remark 1: Similar forms to the matrix inequality (10a) may be found in the literature of master-slave synchronization of Lur'e systems under transmission delays - see [14]. One notes also that the additional constraint (10b) may be solved if the relative degree one assumption $(\operatorname{Rank}(C B)=$ $\operatorname{Rank}(B)$ ) is verified. In order to solve the system given by (10a)-(10b), one may transform it into a convex optimization problem and use an LMI solver feasp, which is well developed in Matlab LMI Toolbox.

Assumption 2: We assume that for any trajectories $z$ of system (3), $\Psi^{k}\left(R_{k} z, u\right)$ is bounded such that $\forall k=1 . . q$, $\exists \mu_{\psi}>0$ such that

$$
\sup _{t \geq 0}\left|\Psi^{k}\left(R_{k} z(t), u(t)\right)\right| \leq \mu_{\psi} .
$$

We assume also that $\theta_{k}$ are bounded in the sense that $\exists \mu_{\theta}>$ 0 such that

$$
\sup _{t \geq 0}\left|\theta_{k}(t)\right| \leq \mu_{\theta}
$$

Assumption 3: We assume that the inputs $u$ are such that for any trajectory $z(t)$ of system (3), $\forall k=1 . . q$, there exist $\mu_{k}, T_{k}>0$, such that $\forall t \geq 0$

$\int_{t}^{t+T_{k}} \Psi^{k}\left(R_{k} z(s), u(s)\right)^{T} B^{T} B \Psi^{k}\left(R_{k} z(s), u(s)\right) d s \geq \mu_{k}$.

Remark 2: In the case where $\Psi^{k}(\cdot)$ doesn't satisfy the boundedness property and if the state is confined to a bounded set $X$, one can always use a smooth bounded saturation function $\sigma: \mathbb{R}^{n} \rightarrow X, x \rightarrow \sigma(x)$ such that $\sigma(x)=x$ to construct a new function $\tilde{\Psi}^{k}(x, u)=\Psi^{k}(\sigma(x), u)$ which is bounded for any $x \in \mathbb{R}^{n}$.
Lemma 1: Let $\Upsilon_{k}(t)$ the solution of the equation (3c), $\forall k=1 . . q$. If $B \Psi^{k}\left(R_{k} z(t), u(t)\right)$ satisfies the assumption 3 , then $\Upsilon_{k}(t)$ is a positive function such that

$$
\forall t \geq \max \left\{T_{k}, k=1 . . q\right\}, \quad \Upsilon_{k}(t) \geq v_{m},
$$

where $v_{m}=\min \left\{\mu_{k} e^{-\alpha T_{k}}, k=1 . . q\right\}$.

Proof: Let $\Upsilon_{k}(t)$ the solution of Equation (3c), $\forall k=$ 1..q. For simplicity, we define

$\Phi^{k}(t):=\Psi^{k}\left(R_{k} z(t), u(t)\right)^{T} B^{T} B \Psi^{k}\left(R_{k} z(t), u(t)\right)$.

We have

$$
\begin{aligned}
\frac{d}{d t}\left(e^{\alpha t} \Upsilon_{k}(t)\right) & =e^{\alpha t}\left(\dot{\Upsilon}_{k}(t)+\alpha \Upsilon_{k}(t)\right) \\
& =e^{\alpha t} \Phi^{k}(t)
\end{aligned}
$$

Integrating the previous equation from 0 to $t+T_{k}$,

$$
\begin{aligned}
e^{\alpha\left(t+T_{k}\right)} \Upsilon_{k}\left(t+T_{k}\right) & =\Upsilon_{k}(0)+\int_{0}^{t+T_{k}} e^{\alpha s} \Phi^{k}(s) d s \\
& \geq \int_{0}^{t+T_{k}} e^{\alpha s} \Phi^{k}(s) d s .
\end{aligned}
$$

Multiplying on both sides by $e^{-\alpha\left(t+T_{k}\right)}$, we obtain

$$
\begin{aligned}
\Upsilon_{k}\left(t+T_{k}\right) & \geq \int_{0}^{t+T_{k}} e^{\alpha\left(s-t-T_{k}\right)} \Phi^{k}(s) d s . \\
& \geq \int_{t}^{t+T_{k}} e^{\alpha\left(s-t-T_{k}\right)} \Phi^{k}(s) d s .
\end{aligned}
$$

For $t \leq s \leq t+T_{k}, e^{-\alpha T_{k}} \leq e^{\alpha\left(s-t-T_{k}\right)} \leq 1$, hence

$$
\Upsilon_{k}\left(t+T_{k}\right) \geq e^{-\alpha T_{k}} \int_{t}^{t+T_{k}} \Phi^{k}(s) d s .
$$

Consequently, using Equation (13), $\forall t \geq T_{k}$, we obtain

$$
\Upsilon_{k}(t) \geq \mu_{k} e^{-\alpha T_{k}} .
$$

Finally, it follows that

$$
\forall t \geq \max \left\{T_{k}, k=1 . . q\right\}, \quad \Upsilon_{k}(t) \geq v_{m},
$$

where $v_{m}=\min \left\{\mu_{k} e^{-\alpha T_{k}}, k=1 . . q\right\}>0$.

Now, we are ready to present our main result.

Theorem 1: Consider the system (1) and the adaptive observer (3). Let the assumptions 1, 2 and 3 hold. Then, the null solution $(e, \tilde{\theta})=(0,0)$ of the error system (4) is globally asymptotically stable for sufficiently small value of the time-delay $h$.

Proof: We apply the Leibniz-Newton formula to the observation error $e(t)$. That is, we have

$$
\begin{aligned}
e(t)-e(t-h) & =\int_{t-h}^{t} \dot{e}(s) d s \\
& =\int_{-h}^{0} \dot{e}(t+\vartheta) d \vartheta .
\end{aligned}
$$

Hence, The dynamics of $e(t)$ can be rewritten as follows

$$
\begin{aligned}
& \dot{e}=(A-K C) e+F \eta(H e, x, u)+K C \int_{-h}^{0} \dot{e}(t+\vartheta) d \vartheta \\
& +B\left(\sum_{k=1}^{q} \bar{\eta}^{k}\left(R_{k} e, x, u\right) \theta_{k}+\sum_{k=1}^{q} \Psi^{k}\left(R_{k} z, u\right) \tilde{\theta}_{k}\right) .
\end{aligned}
$$


Let $\omega(t)=\left(e^{T}(t), \tilde{\theta}^{T}(t)\right)$ and $\omega_{t}$ the function defined in the interval $[-h, 0]$ as

$$
\omega_{t}(\vartheta)=\omega(t+\vartheta), \quad-h \leq \vartheta \leq 0 .
$$

We consider the following Lyapunov-Krasovskii functional:

$$
V\left(t, \omega_{t}\right)=V_{1}(e(t))+V_{2}\left(\omega_{t}\right)+V_{3}(t, \tilde{\theta}(t))
$$

with

$$
\begin{aligned}
V_{1}(e(t)) & =e(t)^{T} P e(t), \\
V_{2}\left(\omega_{t}\right) & =\int_{-h}^{0}(\vartheta+h) \dot{e}(t+\vartheta)^{2} d \vartheta, \\
V_{3}(t, \tilde{\theta}(t)) & =\sum_{k=1}^{q} \rho^{-1} \Upsilon_{k}(t) \tilde{\theta}_{k}(t)^{2},
\end{aligned}
$$

where $\Upsilon_{k}(t)$ is governed by Equation (3c). For simplicity, we introduce the following notations.

$$
\begin{aligned}
& \Gamma(t):=\int_{-h}^{0} \dot{e}(t+\vartheta) d \vartheta \quad ; \quad \beta_{a}:=|A| ; \\
& \beta_{b}:=|B| \quad ; \quad \beta_{c}:=|K C| \text {. }
\end{aligned}
$$

The time derivative of $V_{1}$ along the trajectories of System (4) is given by

$$
\begin{aligned}
& \dot{V}_{1}=e^{T}\left[(A-K C)^{T} P+P(A-K C)\right] e \\
& +2 e^{T} P F \eta(H e, x, u)+2 e^{T} P B \sum_{k=1}^{q} \bar{\eta}^{k}\left(R_{k} e, x, u\right) \theta_{k} \\
& +2 e^{T} P K C \int_{-h}^{0} \dot{e}(t+\vartheta) d \vartheta+2 e^{T} P B \sum_{k=1}^{q} \Psi^{k}\left(R_{k} z, u\right) \tilde{\theta}_{k} .
\end{aligned}
$$

Using (8) and (9), we obtain

$$
\begin{aligned}
& \dot{V}_{1} \leq e^{T}\left[(A-K C)^{T} P+P(A-K C)\right] e \\
& +2 b \eta(H e, x, u) D H e+2 e^{T} P K C \int_{-h}^{0} \dot{e}(t+\vartheta) d \vartheta \\
& -2 \sum_{k=1}^{q} \bar{\eta}^{k T}\left(R_{k} e, x, u\right) \theta_{k} \bar{D}_{k} \bar{\eta}\left(R_{k} e, x, u\right) \\
& +\sum_{k=1}^{q} \bar{b}_{k} \bar{\eta}^{k}\left(R_{k} e, x, u\right) \theta_{k} \bar{D}_{k} R_{k} e \\
& +2 e^{T} P B \sum_{k=1}^{q} \Psi^{k}\left(R_{k} z, u\right) \tilde{\theta}_{k}+2 e^{T} P F \eta(H e, x, u) \\
& -2 \eta^{T}(H e, x, u) D \eta(H e, x, u) .
\end{aligned}
$$

Let us define $\zeta:=\left[e, \eta(H e, x, u), \bar{\eta}^{1}\left(R_{1} e, x, u\right) \theta_{1}, \ldots, \bar{\eta}^{q}\left(R_{q} e, x, u\right) \theta_{q}\right]^{T}$, then one has

$$
\begin{gathered}
\dot{V}_{1} \leq \zeta^{T} \mathcal{S} \zeta+2 e^{T} P K C \int_{-h}^{0} \dot{e}(t+\vartheta) d \vartheta-\varepsilon|e|^{2} \\
+2 e^{T} C^{T} M^{T} \sum_{k=1}^{q} \Psi^{k}\left(R_{k} z, u\right) \tilde{\theta}_{k}
\end{gathered}
$$

where we have also used (10b) and the matrix $\mathcal{S} \leq 0$ is defined above in Assumption 1. Applying the young's inequality $\left|2 c^{T} d\right| \leq \gamma c^{T} c+\frac{1}{\gamma} d^{T} d$ to the term " $2 e(t)^{T} P K C \dot{e}(t+\vartheta)$ " with $c^{T}=e(t)^{T} P K C, d=\dot{e}(t+\vartheta)$ and $\gamma=2$, and integrating from $\vartheta=-h$ to $\vartheta=0$, we obtain

$$
\begin{aligned}
& 2 e(t)^{T} P K C \int_{-h}^{0} \dot{e}(t+\vartheta) d \vartheta \\
\leq & 2 h e(t)^{T} C^{T} K^{T} P^{2} K C e(t)+\frac{1}{2} \int_{-h}^{0} \dot{e}(t+\vartheta)^{2} d \vartheta \\
\leq & 2 h \beta_{c}^{2} p_{M}^{2}|e(t)|^{2}+\frac{1}{2} \int_{-h}^{0} \dot{e}(t+\vartheta)^{2} d \vartheta .
\end{aligned}
$$

hence,

$$
\begin{aligned}
\dot{V}_{1} \leq & -\left(\frac{\varepsilon}{p_{M}}+2 h \beta_{c}^{2} p_{M}^{2} P_{m}^{-1}\right) V_{1}+\frac{1}{2} \int_{-h}^{0} \dot{e}(t+\vartheta)^{2} d \vartheta \\
& +2 e^{T} C^{T} M^{T} \sum_{k=1}^{q} \Psi^{k}\left(R_{k} z, u\right) \tilde{\theta}_{k} .
\end{aligned}
$$

The time derivative of $V_{3}$ along the trajectories of System (4) is given by

$$
\dot{V}_{3}=\rho^{-1} \sum_{k=1}^{q}\left(\dot{\tilde{\theta}}_{k}^{T} \Upsilon_{k} \tilde{\theta}_{k}+\tilde{\theta}_{k}^{T} \Upsilon_{k} \dot{\tilde{\theta}}+\tilde{\theta}_{k}^{T} \dot{\Upsilon}_{k} \tilde{\theta}_{k}\right)
$$

After (3c) and (4b), one obtains

$$
\begin{aligned}
\dot{V}_{3}= & -2 e(t-h)^{T} C^{T} M^{T} \sum_{k=1}^{q} \Psi^{k}\left(R_{k} z, u\right) \tilde{\theta}_{k}-\alpha V_{3} \\
& +\rho^{-1} \sum_{k=1}^{q} \Psi^{k}\left(R_{k} z, u\right)^{T} B^{T} B \Psi^{k}\left(R_{k} z, u\right) \tilde{\theta}_{k}^{2} .
\end{aligned}
$$

Next, applying the young's inequality and Equation (10b), one has

$2 \Gamma^{T} C^{T} M^{T} \sum_{k=1}^{q} \Psi^{k}\left(R_{k} z, u\right) \tilde{\theta}_{k} \leq q \Gamma^{2}+\left(\mu_{\psi} \beta_{b} p_{M}\right)^{2} \sum_{k=1}^{q} \tilde{\theta}_{k}^{2}$,

Using the latter inequality and Equation (20), it follows that

$$
\begin{aligned}
\dot{V}_{3} \leq & v_{m}^{-1}\left(\left(\rho^{-1}+p_{M}^{2}\right)\left(\beta_{b} \mu_{\psi}\right)^{2}-\alpha\right) V_{3} \\
& -2 e^{T} C^{T} M^{T} \sum_{k=1}^{q} \Psi^{k}\left(R_{k} z, u\right) \tilde{\theta}_{k}+q \Gamma^{2}
\end{aligned}
$$

where we have also used positivity of $\Upsilon(t)$-see Remark 2 . On the other hand, the time derivative of $V_{2}$ is given by

$$
\dot{V}_{2}=h|\dot{e}|^{2}-\int_{-h}^{0} \dot{e}(t+\vartheta)^{2} d \vartheta .
$$

From (21) and using the sector condition on $\eta(\cdot)$ and $\bar{\eta}^{k}(\cdot)$, one easily obtains

$$
\begin{aligned}
h|\dot{e}|^{2} \leq & h\left(\beta_{a}+\beta_{c}+b+\sum_{k=1}^{q} \bar{b}_{k} \mu_{\theta}\right)^{2} P_{m}^{-1} V_{1} \\
& +h v_{m}^{-1}\left(\mu_{\psi} \beta_{b}\right)^{2} V_{3}+h \beta_{c}^{2} \Gamma^{2} .
\end{aligned}
$$

Next, using the Jensen's inequality ([15], Lemma 1), it follows that

$$
h \int_{-h}^{0} \dot{e}(t+\vartheta)^{2} d \vartheta \geq\left[\int_{-h}^{0} \dot{e}(t+\vartheta) d \vartheta\right]^{2}=\Gamma^{2} .
$$


Using the above inequalities (25)-(29) and re-arranging terms, the total time derivative of $V\left(t, \omega_{t}\right)$ along the trajectories of System (4) is given by

$$
\begin{aligned}
\dot{V} \leq & {\left[-\frac{\varepsilon}{p_{M}}+h C_{e}\right] V_{1}+\left(q-\frac{N}{2 h}+h \beta_{c}^{2}\right) \Gamma^{2} } \\
& +\left(C_{\theta}+h v_{m}^{-1}\left(\mu_{\psi} \beta_{b}\right)^{2}-\alpha\right) V_{3}
\end{aligned}
$$

where

$$
\begin{aligned}
C_{e} & =p_{m}^{-1}\left(\left(\beta_{a}+\beta_{c}+b+\sum_{k=1}^{q} \bar{b}_{k} \mu_{\theta}\right)^{2}+2 \beta_{c}^{2} p_{M}^{2}\right), \\
C_{\theta} & =v_{m}{ }^{-1}\left(\beta_{b} \mu_{\psi}\right)^{2}\left(\rho^{-1}+p_{M}^{2}\right) .
\end{aligned}
$$

Therefore, if $h$ satisfies the following system of in-equations

we have

$$
\left\{\begin{array}{l}
h C_{e}-\frac{\varepsilon}{2 p_{M}} \leq 0 \\
C_{\theta}+h v_{m}^{-1}\left(\mu_{\psi} \beta_{b}\right)^{2} \frac{\alpha}{2} \leq 0 \\
q-\frac{1}{2 h}+h \beta_{c}^{2} \leq 0
\end{array}\right.
$$

$$
\dot{V}\left(t, \omega_{t}\right) \leq-\frac{\varepsilon}{2 p_{M}} V_{1}(e(t))-\frac{\alpha}{2} V_{3}(t, \tilde{\theta}(t)) \quad \text { a.e. }
$$

Solving for $h$ the system of in-equations (30), we deduce that (31) holds for

$$
h \leq \min \left\{\pi_{a}, \pi_{b}, \pi_{c}\right\}
$$

where $\pi_{a}=C_{e}^{-1}\left(\frac{\varepsilon}{2 p_{M}}\right), \pi_{c}=\frac{-q+\sqrt{q^{2}+2 \beta_{c}^{2}}}{2 \beta_{c}^{2}}$ and $\pi_{b}=v_{m}\left(\mu_{\psi} \beta_{b}\right)^{-2}\left(\frac{\alpha}{2}-C_{\theta}\right)$.

In summary, we have:

i) $V\left(t, \omega_{t}\right)$ is positive definite and radially unbounded,

ii) $\dot{V}\left(t, \omega_{t}\right)$ is negative definite,

hence, invoking the Lyapunov-Krasovskii Stability Theorem ( [16], Theorem 4.1), we conclude that the null solution $(e, \tilde{\theta})=(0,0)$ (i.e $w=0)$ of the error system (4) is globally asymptotically stable, which completes the proof.

It is to be noticed, that the same design procedure of the proposed observer still valid when the measurement delay is time-varying and bounded and it may be shown that for sufficiently small value of the delay upper-bound, both state and unknown parameters estimation are ensured. We note, also, that the design constants $\alpha$ and $\rho$ may be suitably selected in such away that the interval of admissible values of the time-delay $h$, as given by the constraint (32), is enlarged.

\section{NUMERICAL EXAMPLES}

\section{A. Nonlinear Mass-Spring-Damper}

Consider the uncertain mass-spring-damper system

$$
\begin{aligned}
& \dot{x_{p}}=x_{v}, \\
& \dot{x_{v}}=-\frac{\bar{k}}{\bar{m}} x_{p}+\frac{1}{\bar{m}} u(t)-\frac{1}{\bar{m}}\left|x_{v}\right| x_{v} \bar{b} .
\end{aligned}
$$

where $p \in \mathbb{R}$ is the position and $v$ is the velocity. The constants $\bar{k}=0.5$ and $\bar{m}=1$ represent respectively spring stiffness and the mass. The damping coefficient $\bar{b}=1$ is is the unknown parameter to be estimated. It is assumed also that the sum of the position and velocity is measured and subject to a known constant delay $h=0.03 s$. This system is of the form (1) with

$$
\begin{gathered}
x=\left[\begin{array}{l}
x_{p} \\
x_{v}
\end{array}\right], A=\left[\begin{array}{cc}
0 & 1 \\
-0.5 & 0
\end{array}\right], B=\left[\begin{array}{c}
0 \\
-1
\end{array}\right], \\
F=\left[\begin{array}{l}
0 \\
1
\end{array}\right], C=\left[\begin{array}{ll}
1 & 1
\end{array}\right], H=\left[\begin{array}{ll}
0 & 1
\end{array}\right], \\
f(H x, u)=u(t)=\sin (2 \pi t)+10 \sin \left(\pi t+\frac{\pi}{2.5}\right), k=1, \\
R_{1}=\left[\begin{array}{ll}
0 & 1
\end{array}\right], \Psi^{1}\left(R_{1} x, u\right)=\left|x_{v}\right| x_{v}, \text { and } \theta=1
\end{gathered}
$$

We design an observer for (33) as given by (3) $(N=2)$ . Solving the system (10) given in Assumption (1), one obtains $D=\bar{D}_{1}=1.6905, P=\left[\begin{array}{cc}10.1536 & 1.8658 \\ 1.8658 & 1.8658\end{array}\right]$,
$\varepsilon=3.0818$.

We deduce also the numeric values of the observer matrices: $K=\left[\begin{array}{c}-0.3136 \\ 3.4536\end{array}\right], M=-1.8658, \rho=10, \alpha=10$.

We initialize the state $x(t)$ of System $(33)$ at $x_{0}=[0,0]^{T}$, $\forall s \in[-0.015,0]$. The observer are started with initial conditions $z(s)=[0.1,0.1]^{\top}, \forall s \in[-0.015,0], \hat{\theta}=1.5$, $\Upsilon=0.2$. The simulation results are as follows. Figure 1 illustrates the convergence of the observation errors and figure 2 shows that the unknown parameter is well recovered by the proposed adaptive observer.

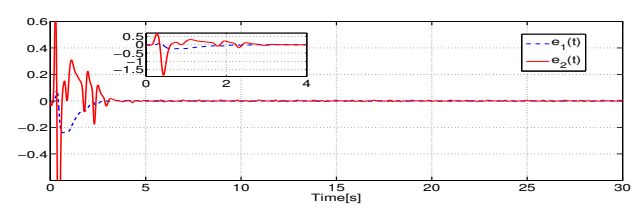

Fig. 1. Estimation errors $e_{1}=x_{p}-z_{p}$ and $e_{2}=x_{v}-z_{v}$ in the presence of a constant observation delay

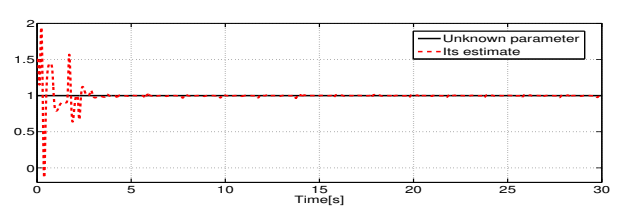

Fig. 2. The unknown parameter $\theta$ and its estimate in presence of a constant observation delay

B. a chaos-based communication system with a transmission time-delay using a "Duffing" oscillator

We consider a chaotic "Duffing" oscillator. A digital information $m(t)$ is injected in its dynamics to modulate one of its parameters. The output $y$ is corrupted by a known and constant transmission delay $h=0.08$. Note that in this 
application, $m(t)$ plays the role of the unknown parameter. Then, the dynamics of the chaotic transmitter is given by

$$
\begin{aligned}
\dot{X}_{1} & =X_{2}, \\
\dot{X}_{2} & =-0.4 X_{2}-1.1 X_{1}-(1+m(t)) X_{1}^{3}+\cos (1.8 t) \\
y(t) & =X_{1}(t-h)+X_{2}(t-h) .
\end{aligned}
$$

which is obviously of the form (1) with

$$
\begin{gathered}
x=\left[\begin{array}{l}
X_{1} \\
X_{2}
\end{array}\right], A=\left[\begin{array}{cc}
0 & 1 \\
0.4 & -1.1
\end{array}\right], B=\left[\begin{array}{c}
0 \\
-1
\end{array}\right], \\
F=\left[\begin{array}{c}
0 \\
-1
\end{array}\right], C=\left[\begin{array}{ll}
1 & 1
\end{array}\right], H=\left[\begin{array}{ll}
1 & 0
\end{array}\right], \\
f(H x, u)=X_{1}^{3}+u, u(t)=\cos (1.8 t), k=1, \\
R_{1}=\left[\begin{array}{ll}
1 & 0
\end{array}\right], \Psi^{1}\left(R_{1} x, u\right)=X_{1}^{3}, \text { and } \theta(t)=m(t)
\end{gathered}
$$

The receiver is given by (3). Solving the system (10) given in Assumption (1), one obtains

$$
\begin{aligned}
& D=\bar{D}_{1}=2.6464, P=\left[\begin{array}{cc}
12.1437 & 1.5679 \\
1.5679 & 1.5679
\end{array}\right], \\
& \varepsilon=5.9542 .
\end{aligned}
$$

We deduce also the numeric values of the observer matrices:

$K=\left[\begin{array}{l}0.0596 \\ 3.4896\end{array}\right], M=-1.5679, \rho=10, \alpha=0.5$.

The state $x(t)$ of System $(34)$ is initialized at $x_{0}=[0,0]^{T}$, $\forall s \in[-0.04,0]$. The observer is started with initial conditions $z(s)=[1,2]^{\top}, \forall s \in[-0.04,0], \hat{m}=0.6$, $\Upsilon=0.2$. The simulation results are as follows. Figure 3 illustrates the synchronization between the transmitter and the receiver and figure 4 shows that the transmitted information is well recovered by the receiver based on the proposed adaptive observer.

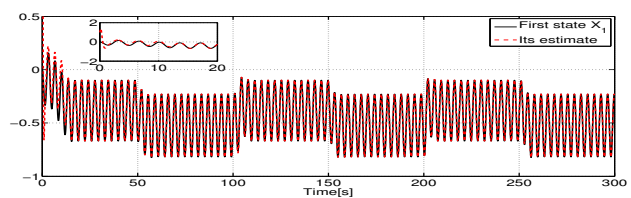

Fig. 3. The first state $X_{1}$ and its estimate in the presence of a constant transmission delay

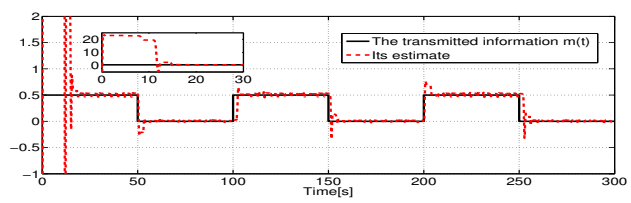

Fig. 4. The transmitted information $m(t)$ and its estimate in presence of a constant transmission delay

\section{CONCLUSION}

In this work, we propose an adaptive observer for a class of nonlinear systems with slope restricted nonlinearities, unknown parameters and delayed outputs. Both state estimation and parametric convergence are shown to be ensured for sufficiently small values of the time-delay and if a condition of persistent excitation is satisfied. For illustration, the approach was evaluated through a nonlinear mass-spring-damper system application and a chaos-based communication system subject to a transmission time-delay. In a future work, we will investigate the extension of the proposed approach to the case where the time-delay takes large values, using cascade observers.

\section{REFERENCES}

[1] M. Boutayeb, "Observer design for linear time-delay systems," Syst. \& Contr. Letters, vol. 44 (2), pp. 103-109, 2001.

[2] G. Besanc̀on, D. Georges, and Z. Benayache, "Asymptotic state prediction for continuous-time systems with delayed input and application to control," European Control Conference, pp. 1786-1791, 2001.

[3] A. Germani, C. Manes, and P. Pepe, "A new approachto state observation of nonlinear systems with delayed output," IEEE Trans. on Automat. Contr., vol. 47 (1), pp. 96-101, 2000.

[4] N. Kazantzis and R. A. Wright, "Nonlinear observer design in the presence of delayed output measurements," Sys.\& Control Letters, vol. 54, pp. 877-886, 2005.

[5] K. Subbarao and P. C. Muralidhar, "A state observer for lti systems with delayed outputs: Time-varying delay," in American Control Conference, (Washington, USA), pp. 3029-3033, 2008.

[6] F. Cacace, A. Germani, and C. Manes, "An observer for a class of nonlinear systems with time varying observation delay," Sys.\& Control Letters, vol. 59, pp. 305-312, 2010.

[7] T. Ahmed-Ali, E. Cherrier, and M. Msaad, "Cascade high gain observers for nonlinear systems with delayed output," in Proc. 48th. IEEE Conf. Decision Contr., (Shanghai, China), pp. 8226 -8231, 2009.

[8] V. V. Assche, T. Ahmed-Ali, C. A. B. Hann, and F. LamnabhiLagarrigue, "High gain observer design for nonlinear systems with time varying delayed measurements," in 18th IFAC World congress, (Milano, Italia), pp. 692-696, 2011.

[9] G. Besançon, "Remarks on nonlinear adaptive observer design," Systems and Control Letters, vol. 41, pp. 271-280, 2000.

[10] Q. Zhang, "Adaptive observers for mimo linear time-varying systems," IEEE Trans. on Automat. Contr., vol. 47, pp. 525-529, 2002.

[11] A. Xu and Q. Zhang, "Nonlinear system fault diagnosis based on adaptive estimation," Automatica, vol. 40, pp. 1183-1193, 2004.

[12] A. Loria, E. Panteley, and A. Zavala, "Adaptive observers with persistency of excitation for synchronization of chaotic systems," IEEE Trans. on Circ. Syst. I: Regular Papers, vol. 56, no. 12, pp. 2703-2716, 2009.

[13] M. Arcak and P. Kokotović, "Observer-based control of systems with slope-restricted nonlinearities," IEEE Trans. on Automat. Contr. vol. 46, no. 7, pp. 1146-1150, 2001.

[14] Q. L. Han, "On designing time-varying delay feedback controllers formaster-slave synchronization of lur'e systems," IEEE Trans. on Circ. Syst. I: Regular Papers, vol. 54, pp. 1573-1583, 2007.

[15] L. da Cruz Figueredo, J. Ishihara, G. Borges, and A. Bauchspiess, "New delay-and-delay-derivative-dependent stability criteria for systems with time-varying delay," in Proc. 49th. IEEE Conf. Decision Contr., (Atlanta, USA), pp. 1004-1009, 2010.

[16] A. Loria, F. Lamnabhi-Lagarrigue, and E. Panteley, Advanced topics in control systems theory. London, ISBN: 1-84628-313-2.: Series Lecture Notes in Control and Information Science, Springer Verlag, 2005. 